\title{
An Embedded System for Practical Security Analysis of Contactless Smartcards
}

\author{
Timo Kasper, Dario Carluccio, and Christof Paar \\ Communication Security Group, \\ Ruhr-University Bochum, Germany \\ \{tkasper, carluccio, cpaar\}@crypto.rub.de \\ www.crypto.rub.de
}

\begin{abstract}
ISO 14443 compliant smartcards are widely-used in privacy and security sensitive applications. Due to the contactless interface, they can be activated and read out from a distance. Thus, relay and other attacks are feasible, even without the owner noticing it. Tools being able to perform these attacks and carry out security analyses need to be developed. In this contribution, an implementation of a cost-effective, freely programmable ISO 14443 compliant multi function RFID reader and fake transponder is presented that can be employed for several promising purposes.
\end{abstract}

Keywords: RFID, Low Level Reader, Fake RFID Tag, Relay Attack.

\section{Introduction}

As technology evolves and chip sizes decrease, RFID (Radio Frequency Identification) is becoming widely-used for ubiquitous tasks. The ISO 14443 [14] norm for contactless smartcards is currently employed in various security sensitive applications, such as the electronic passport [3] to store biometric data and RFID-enabled credit cards 31. The contactless interface brings new opportunities for potential attackers: The device can not only be activated and read out without the actual owner taking note of it, but also can the transmission of data via the RF (Radio Frequency) field be eavesdropped from a distance of several meters 8. This demanded for countermeasures, such as encryption of the interchanged data and the BAC (Basic Access Control) in the electronic passport [15].

New Perils. However, modern attackers get physical access to the chip or its electromagnetic field and perform so called side channel attacks like a DEMA (Differential Electro Magnetic Analysis) which can be performed with contactless smartcards 5]. By measuring and evaluating the electromagnetic emanation and correlating it with the code running on the chip, information about a secret key stored on it is gathered. A remote power analysis was performed by Oren and Shamir [22]. Their attack, targeting at RFID tags operating in the UHF (Ultra High Frequency) range, could probably also be applied to contactless smartcards. 
Furthermore, fault injection 11 in order to cause a malfunction of the device may reveal a clue to the secret key [2]. A relay attack is also feasible [11: By redirecting the data interchanged between a reader and a tag over a separate communication channel in real time, one can pretend to be the owner of someone else's tag.

The industry wants to keep the prices low and, due to the restricted energy supply of the chip via the RF field, the number of switching transistors is limited [19]. Hence, security measures and physical protection on the chin 2 may be very lightweight or won't be employed at all [29, even when security or privacy issues are relevant.

Towards More Security. As fraud involving contactless smartcards is becoming more profitable, soon the first real world offences are expected to emerge. To test and then improve the security of the existing systems, tools being able to perform attacks, as well as to analyse the capabilities and functionality of the used hardware and protocols, need to be developed. As the standards differ very much with regard to operating frequency, communication interface and transmission protocol 9, the hardware for a reasonable security analysis must be custom-made and tailored to a particular one. We opted for the ISO 14443, being the most common and widespread norm for contactless smartcards.

Our Contribution. A cost-efficient embedded system shall be developed to ease the security analysis of, maybe cryptographically enabled, smartcards with an ISO 14443A compliant RF interface. Extensive control of the communication and the energy supply is demanded, as well as interoperability with other hardware and measurement equipment. In addition, stand-alone operation is required for performing practical attacks and mobile data acquisition. Some of the tasks to be made possible are

- communication on the bit layer with a low level reader,

- emulation of an ISO 14443 compliant tag,

- perform practical replay and man-in-the-middle (relay) attacks,

- assist remote power analysis, DEMA and fault injection analysis,

- acquisition and logging of the interchanged data, and

- testing of different types of antennas and power amplifiers.

\section{ISO 14443 RFID Operation Principle}

As depicted in Fig. 1, a minimum RFID system consists of two main components, namely a reader generating a sinusoidal field with a carrier frequency of $f_{c}=13.56 \mathrm{MHz}$ which supplies the second component of the system, a tag or transponder, with energy and often a clock. Both devices are equipped with a coupling element which in the case of the ISO 14443 is a coil with typical 3-10 windings, allowing for data transfer in both directions.

\footnotetext{
${ }^{1}$ For instance by manipulating the energy supply or by emission of laser pulses.

${ }^{2}$ Including masking and sensors for detecting fault injection or light.
} 

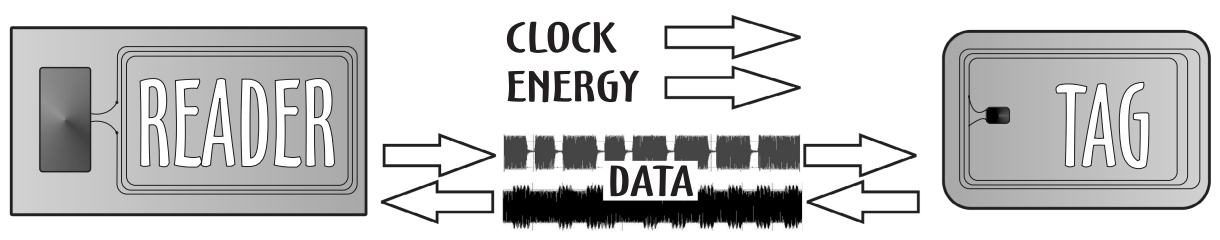

Fig. 1. RFID Operation Principle

The wavelength $\lambda=\frac{c}{f}$ of the electromagnetic field, where $c$ denotes the speed of light and $f$ the carrier frequency, is approximately $22.1 \mathrm{~m}$ at $13.56 \mathrm{MHz}$ and therefore several times greater than the typical operating distance of 8 $15 \mathrm{~cm}$ between reader and tag. Accordingly, the field emitted from the coi 3 of the reader may be treated as purely magnetis, leading to the term inductive coupling for describing the communication and energy link between reader and $\operatorname{tag}[9]$.

Reader $\rightarrow$ Tag. The reader sends data to the tag using a modified (pulsed) Miller code 9]. Pauses have to be created with a duration of approximately $2.5 \mu \mathrm{s}$ with $100 \%$ ASK (Amplitude Shift Keying), i.e., the field has to be completely switched off and on by the reader (compare with the upper waveform in Fig. 11).

Tag $\rightarrow$ Reader. Due to the inductive coupling, the feedback of the transponder drawing more or less energy from the field can be sensed on the side of the reader. Hence, the tag transmits data by switching on and off an additional load and thereby deliberately drawing more energy from the field than during normal operation. This process is termed load modulation. As the coupling between tag and reader is pretty weak, the resulting effect on the field is almost not noticeable (compare with the lower waveform in Fig. 11). For this reason, a subcarrier of the frequency of the reader is used for the load modulation, resulting in the transmitted information being placed in sidebands and so making its detection possible 9. The data is transmitted employing Manchester code and synchronously to the field of the reader, utilising the described OOK (On-Off Keying) with a subcarrier of $\frac{f_{c}}{16}=847.5 \mathrm{kHz}$.

\section{Implementation Details of the Embedded System}

The developed embedded system consists of a multi purpose reader device which is equipped with a $\mu \mathrm{C}$ (microcontroller), an $\mathrm{RF}$ interface and some components for signal processing. A second device, termed fake tag, is designed to appear like an authentic tag to an RFID reader and furthermore can acquire the information contained in the field. Between the two units, a communication link can be established.

\footnotetext{
3 The technical term for coil is inductivity.

${ }^{4}$ Similar to the common transformer principle.
} 


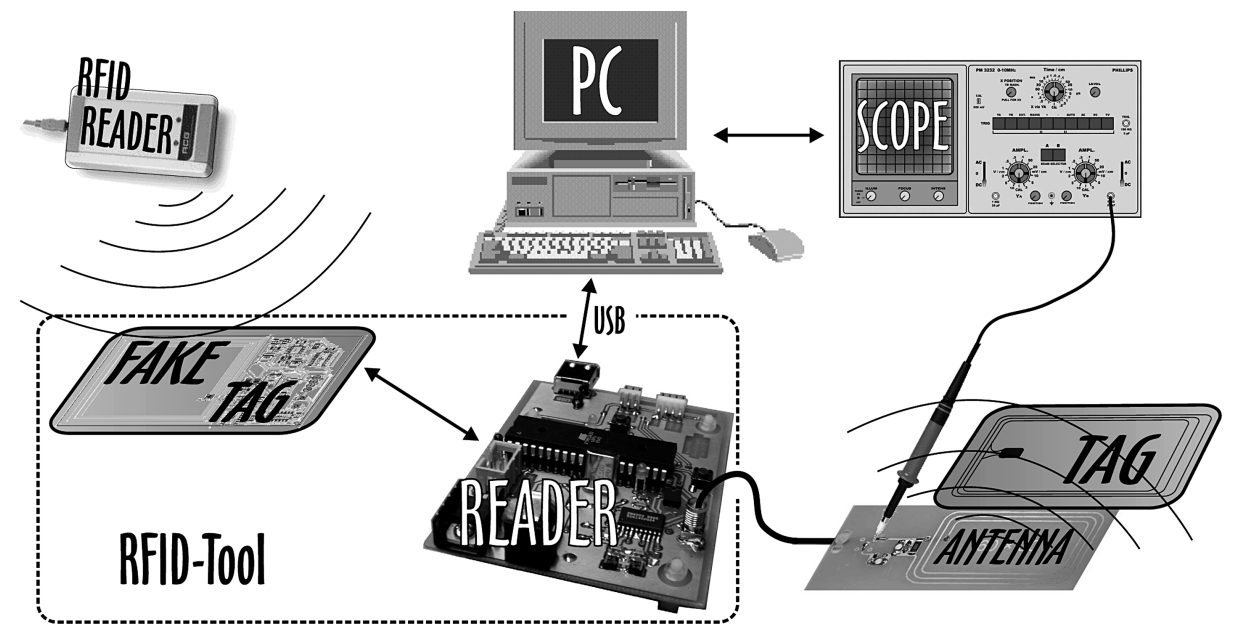

Fig. 2. System Overview

As depicted in Fig. 2, the RFID tool is effortlessly integrated in a measurement setup consisting of a PC (Personal Computer), the developed reader and fake tag, a digital oscilloscope and more equipment for measuring and inducing faults. The PC controls the measurements and later combines and further processes the data acquired from scope and reader. This work focuses on ISO 14443 type A devices using a data rate of $106 \frac{k B i t}{s}$, as specified in the standard [14.

\subsection{Reader}

The operation principle of the low level reader, as detailed in this section, is depicted in Fig. 3. The RFID tool is based on an Atmel ATMega32 [1 microcontroller, clocked at $13.56 \mathrm{MHz}$, which is amongst others equipped with $32 \mathrm{kB}$ Flash RAM, 1 kB non-volatile EEPROM(Electronically Erasable Programmable Read Only Memory) and an ADC (Analog to Digital Converter). For flexible operation and testing, the software running on the $\mu \mathrm{C}$ can be updated through a PC without the need to remove it from the PCB (Printed Circuit Board).

The main part of the analogue front end is provided by the EM 4094 RFtransceiver [6] which possesses a $200 \mathrm{~mW}$ push pull transmitter operating at $13.56 \mathrm{MHz}$, is capable of $100 \%$ ASK and ready for ISO $14443 \mathrm{~A}$ operation at a price of less than $5 €$. The received HF-Signal can be conditioned by internal filters and adjustable receiver gain. The chip allows for transparent operation, i.e., a high input level on its DIN pin will instantly switch off the field, while a low level switches it on, thus enabling flexible, direct control of the RF field. The output stage of the transceiver has been matched for feeding the signal into 


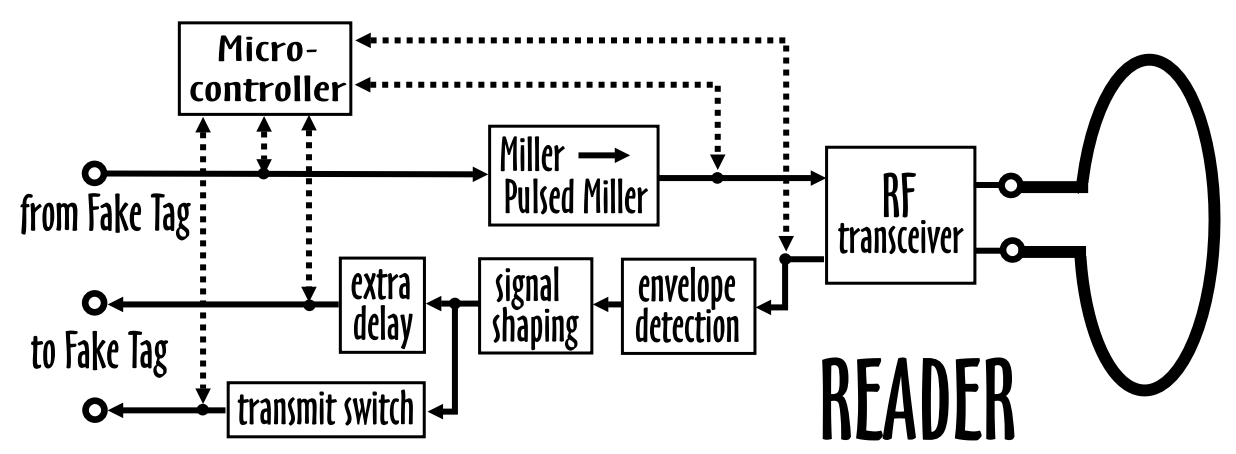

Fig. 3. Operation Principle of the RFID-Reader

a common $50 \Omega$ coaxial cable, so that different antennas and power amplifiers can be connected to a socket placed on the PCB.

Fast communication with a PC or other USB (Universal Serial Bus) equipped hardware is made possible by the FT 245R 10 parallel to USB chip from FTD 5 . The device allows for receiving or sending of packets of eight data bits by pulling a read or write input pin high and low. Using the supplied VCP (Virtual Com Port) driver, a maximum data transfer rate of $1 \frac{M B y t e}{s}$ is possible, while the USB port appears as a standard serial COM port, so that a reliable communication can be established fairly easy.

To disburden the $\mu \mathrm{C}$, the creation of pauses (see Sect. 2) is sourced out to a 74123 26] monoflop, creating the required pulses on every rising edge emitted by the $\mu \mathrm{C}$. These are fed into the EM 4094 transceiver, resulting in the field being switched off shortly. Two more monoflops, creating pulses on any type of transition, convert Miller coded data, for instance received from the fake tag during a relay attack, to pulsed Miller coded data which is again applied to the field.

The modulated Manchester code, output by the EM 4094, is demodulated using an envelope detector circuit. The signal is rectified by a diode and then fed into a LPF (Low Pass Filter). An LM 311 [21] voltage comparator decides whether the subcarrier is present or not, resulting in Manchester encoded data with the appropriate 0 and $5 \mathrm{~V}$ levels at its output.

As the demodulation of the signal received from the RF transceiver costs some time (in this case $\approx 1.5 \mu \mathrm{s}$ ), it can happen, that the answer of the tag is not well synchronised with the reader when relaying data. To take this into account, a circuit has been developed for adding a short adjustable time delay to the outgoing signal, without altering its waveform.

An interface for serial communication between the developed reader and fake tag is also installed on the PCB. The data pins can be driven directly by the peripheral circuitry of the RFID tool or steered by the $\mu \mathrm{C}$, which allows for the emulation of a tag as well as for $\mu \mathrm{C}$-based processing of the interchanged signals.

$\overline{{ }^{5} \text { www.ftdichip.com }}$ 


\subsection{Fake Tag}

The counterpart to the reader, named fake tag, can be utilised for relay attacks as well as for stand-alone emulation of a contactless smartcard. Its functional principle is depicted in Fig. (4. Unlike a normal (passive) tag, the fake tag described here has its own power supply $\sqrt{6}$ which may also be used for supplying a radio module for communicating with the reader.

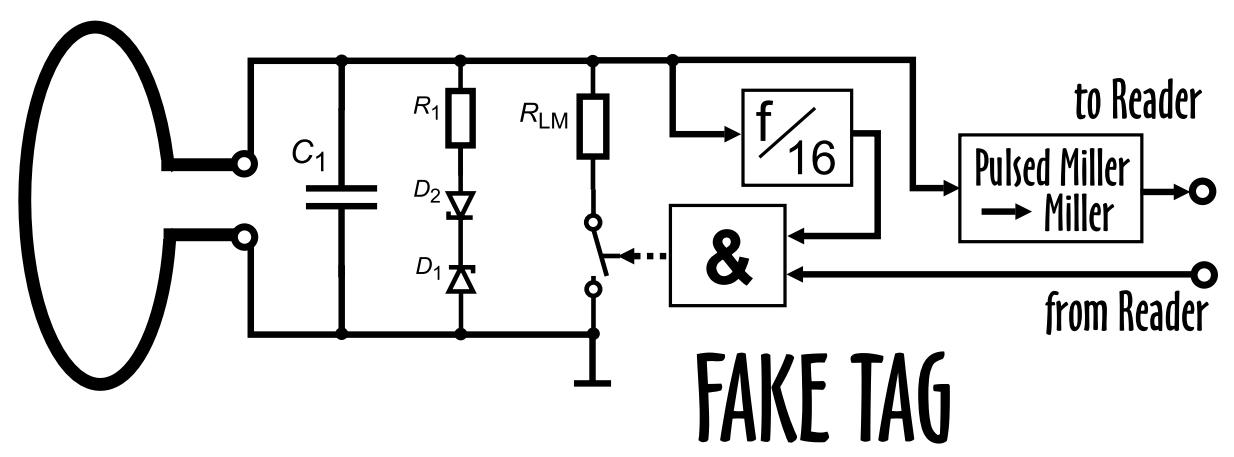

Fig. 4. Operation Principle of the Fake-Tag

A tag needs a coil to establish the coupling with its counterpart at the reader (see Sect. 2). A capacitor is connected in parallel to this inductance, to form a parallel resonant circuit. For an ideal parallel resonant circuit, $f_{c}=\frac{1}{\sqrt{L C}}$ applies [32, where $f_{c}$ denotes the carrier frequency of the reader, $C$ the capacitance and $L$ the inductance of the tuned circuit. In practice, first the value for $L$ is derived from the shape and dimensions of the coil. Afterwards, the optimal $C$ is calculated and then realised as a variable capacitor, so that the circuit can be tuned more precisely later on. The induced voltage can become relatively large, so two antiparallel Zener-diodes limit the maximum possible voltage and thus protect the rest of the circuit.

The subcarrier with a frequency of $\frac{f_{c}}{16}=847.5 \mathrm{kHz}$ is derived from the field generated by the reader. For this, the antenna is connected to the input of a 4-bit binary counter 74393 23 through a resistor which limits the maximum current into the input stage, as proposed in [7. The fourth output of the binary counter toggles every $2^{3}=8$ clock cycles which equals frequency division by 16 , i.e., the desired subcarrier. For modulating the incoming Manchester coded signal with the subcarrier, a 740824 AND gate combines it with the output of the binary counter.

To achieve the load modulation, as described in Sect. 2, a resistor has to be connected in parallel to the coil of the tag. This is realised via an IRFD 11013 $\mathrm{N}$-channel MOSFET, allowing for fast switching and a maximum drain-source voltage of $100 \mathrm{~V}$. The output of the AND gate (see above) is connected to the gate of the transistor. Accordingly, by toggling the resistor, the $848 \mathrm{kHz}$-modulated

\footnotetext{
${ }^{6}$ Can be a small lithium battery.
} 
Manchester code is in turn modulated onto the $13.56 \mathrm{MHz}$ field of the reader and the information put into the sidebands of the carrier.

To acquire data from a nearby reader, an LM 311 comparator combined with two envelope detectors (as detailed in Sect. 3.1) are connected in parallel to the resonant circuit. One of the detectors has a fast response time and distinguishes between the field being completely switched off and the load modulation case. The other envelope detector reacts slower and averages the signal at the antenna, for adapting the threshold voltage of the comparator to the current field strength. This approach immunises the circuit to noise caused by the RF field and so extends the operating range.

The output of the comparator is connected to a 7474 D-type flip-flop 25], whose inverted output is fed back into its input. Hence, a change of the output state occurs on every rising edge at the input. This conversion from pulses into transitions, resulting in a Miller coded data signal, is amongst others necessary to reduce the bandwidth required for the communication link of the RFID tool.

\subsection{Operation Modes}

The software for the $\mu \mathrm{C}$ is mainly written in $\mathrm{C}$, with assembler code inserted, where the execution speed is crucial. Besides, a $\mathrm{C}$ library for controlling the RFID tool from a PC, as well as a corresponding GUI (Graphical User Interface) is available. The following operating modes are currently implemented:

- bit level reader: the reader is freely controlled by the PC via USB,

- stand-alone reader: mobile operation with an arbitrary command sequence prestored in the EEPROM (and acquired data stored into it),

- tag emulation: the fake tag is directly controlled via USB,

- mobile tag emulation: prestored data is replayed by the fake tag, while the reader's requests are recorded to the EEPROM,

- relay mode: mobile operation of both reader and fake tag, while the relayed bits in both directions can be recorded to the EEPROM.

Further routines are provided for generating ISO 14443 compliant bitstreams and for reading and writing the non-volatile EEPROM.

\section{Results}

The flexible low level reader mode has been successfully tested with several ISO 14443 compliant tags which are partly listed below in this section. The exact behavior and timing of the contactless interface can be flexibly steered, even transcending the ISO standard, if desired.

The data sent out by the fake tag is accepted by an ACG7 Dual 2.1 Passport Reader in our laboratory, just as if it was a genuine tag. During our tests, the answer of the fake tag to a request issued by the reader was intentionally delayed

\footnotetext{
${ }^{7}$ http://acg-id.aaitg.com
} 
by more than $250 \mu$ s and the resulting behaviour was analysed. Compliance of the ACG reader with the strict timing requirements during the initialisation phase 8 could not be observed, i.e., the delayed answer was still accepted, thus easing relay attacks.

The RFID tool can be used for logging the data interchanged in any direction. This can be helpful to analyse unknown protocols, as well as for further processing, e.g., key-search with cost effective hardware, such as proposed in [18.

Various antennas were built, tuned to resonance with the carrier frequency and matched to a $50 \Omega$ coaxial cable, to perform tests with regard to the operating range and the influence of the physical environment of the card.

For executing a relay attack [16], the antenna of the bit level reader possessed by the offender has to be placed close enough to a contactless card of the victim. At the same time, the fake tag is brought into the field of an RFID reader, e.g., at the cash desk, by an accomplice. The data being transferred by this reader is acquired and directly forwarded on the bit layer through a communication link to the attacker. There, the data is retransmitted to the card of the victim. Its answer is relayed back to the reader at the cashpoint and so, as the attackers continue relaying the data, both reader and tag will be convinced that they are in close vicinity to each other and thus carry out their task, e.g., authorise a payment.

Such an attack has been successfully carried out using the here described embedded tool with

- an RFID-enabled passport, issued by the Federal Republic of Germany,

- a student identity chip card of the Ruhr-University in Bochum, Germany,

- Philips classic Mifare and DESFire cryptographically enabled smartcards,

- an Atmel AT88SC153 smartcard, and

- a ticket for the FIFA world cup 2006 in Germany,

until to at least reading out the UID (Unique Identifier) of the tags. In the case of the Mifare classic, after a the successful login, encrypted data blocks were read out and modified remotely. Furthermore, the 64 Byte content of a world cup ticket was read out using the relay mode and the interchanged data was recorded for subsequently analysing the protocol. The Philips Mifare Ultralight chip embedded in the ticket 28] provides no encryption at all. Hence, the RFID access control could easily be spoofed with the developed embedded system, by means of a replay attack, as the communication protocol is fully published in the data sheet [27].

When relaying data, a delay is inevitable, as described in Sect. 3.1. However, if a reader scrutinised the timing, a relay attack could still be carried out successfully, as the (fixed) bit sequence of a command could be stored in the $\mu \mathrm{C}$ and sent out instantly after an incoming request.

Hancke and Kuhn 12 proposed a countermeasure for relay attacks, based on ultra-wideband pulses. Still, as it is not employed in current tags, the most effective way to enhance privacy is constructing a Faraday's cage for the tag: Our experiments proved, that a single layer of aluminum foil wrapped around

\footnotetext{
${ }^{8}$ ISO 14443 requires the tag to answer to a $R E Q A$ exactly after $86.9 \mu \mathrm{s}$.
} 
the smartcard completely protects it from being activated or read out by an unauthorised reader.

The implemented embedded system has become a valuable part of the measurement setup in our laboratory and is currently employed to assist several ongoing security analyses (compare with Sect. 56).

\section{$5 \quad$ Future Prospects}

At the moment, the achieved read range with the developed reader and the antennas used is approximately $5-10 \mathrm{~cm}$. It is possible to extend this range to $25 \mathrm{~cm}$ [17, using a power amplifier 20, and a large copper tube antenna 30.

The communication protocol of a Philips Mifare DESFire contactless smartcard has been reverse engineered until to the point necessary for carrying out a DEMA [4. In the respective attack, the challenges 9 were generated by a proprietary RFID reader and had to be extracted from the oscilloscope waveforms, which meant a severe, time consuming constraint for the analysis. Using the developed system, arbitrary access to the contactless interface is provided, allowing amongst others for freely chosen challenges. A DEMA is based on a statistical test at one certain point in time, so subsequent measurements need to be synchronised before superimposing them. The for his purpose required reliable signal to trigger the oscilloscope can now also be emitted by the RFID tool, thus further improving the attack.

It is promising to use the embedded system for execution of a remote power analysis. During the pauses occurring in the field of the reader (compare with Sect. 2), a tag draws its energy from a built-in capacitor which recharges, when the field is activated again. Consequently, different shaped energy peaks emerge in the field, depending on the amount of power consumed by the tag during the energy gap. This behaviour might be exploited to derive a secret key stored on the tag. The RFID tool provides a corresponding output signal which can be acquired by the Atmel's internal ADC or an oscilloscope.

As the reader can be arbitrarily programmed, fault injection attacks are feasible [2] in which the device is forced to show erroneous performance, by perturbing physical parameters like the power supply or the clock frequency. Furthermore, controlling of external pulse generators and other fault injection equipment with the RFID tool is possible.

Finally, any new protocols based on the ISO 14443 standard can be implemented and tested. If additional hardware was required, it could easily be connected to the PCB.

\section{Conclusion}

In this contribution, we present an embedded implementation of a cost effective, arbitrarily programmable RFID reader and a fake tag which can be used for various promising purposes. The tool was built using electronic hobbyist equipment

\footnotetext{
${ }^{9}$ Random numbers interchanged for the authentification.
} 
and off the shelf components at a cost of less than $40 €$, and its design is simple enough to be reproduced by a low skilled attacker. With the developed hardware, we have successfully carried out relay and replay attacks between various contactless smartcards and a commercial RFID reader. Integrated in a measurement system, the proposed tool can help to carry out security analyses, such as a DEMA or a remote power analysis, and assist fault injection attacks. The stand-alone operation modes permit for mobile tag emulation, reader operation and logging of the interchanged data.

Employing ISO 14443 compliant contactless smartcards in security sensitive applications should be regarded very critically, as the physical interface is proven to be insecure against relay attacks. A smartcard identified by a reader does not have to be in its direct vicinity, as declared by many manufacturers. Instead, the data can be forwarded from large distances without permission or even notification of the owner, as described in this paper, with little effort. If an RFID tag is indispensable, we suggest a metal shielding to prevent unauthorised access and propose that the card should not be able to become active, unless the owner has performed an action, e.g., press a button or open the cover of his electronic passport.

\section{References}

1. Atmel. ATMega32 data sheet. http://www.atmel.com/dyn/resources/prod_documents/doc2503.pdf.

2. E. Biham and A. Shamir. Differential fault analysis of secret key cryptosystems. Lecture Notes in Computer Science, 1294:513-, 1997.

3. BSI - German Ministry of Security. ePass - Der Reisepass mit biometrischen Merkmalen. http://www.bsi.de/fachthem/epass/.

4. D. Carluccio. Electromagnetic Side Channel Analysis for Embedded Crypto Devices. Master's thesis, Chair for Communication Security at the Ruhr University Bochum, 2005. Diploma thesis.

5. D. Carluccio, K. Lemke, and C. Paar. Electromagnetic side channel analysis of a contactless smart card: first results. In ECRYPT Workshop on RFID and Lightweight Crypto, pages 44-51, Graz, Austria, July 2005. ECRYPT. http://www.iaik.tu-graz.ac.at/research/krypto/events/RFID-Slidesand Proceedings/Proceedings-WSonRFIDandLWCrypto.zip

6. EM Microelectronic. EM4094 fact sheet. http://www.emmicroelectronics.com/ webfiles/product/rfid/ds/EM4094_fs.pdf

7. Fairchild Semiconductors. Application note 313: DC electrical characteristics of MM74HC high speed logic. http://www.fairchildsemi.com/an/AN/AN-313.pdf.

8. T. Finke and H. Kelter. Radio Frequency Identification Abhörmöglichkeiten der Kommunikation zwischen Lesegerät und Transponder am Beispiel eines ISO14443Systems. http://www.bsi.de/fachthem/rfid/Abh_RFID.pdf. BSI - German Ministry of Security.

9. K. Finkenzeller. RFID Handbook: Fundamentals and Applications in Contactless Smart Cards and Identification. John Wiley and Sons, 2nd edition, 2003.

10. FTDI. FT245 USB chip data sheet. http://www.ftdichip.com/Documents/ DataSheets/DS_FT245R_v105.pdf

11. G. Hancke. A practical relay attack on ISO 14443 proximity cards. http://www.cl.cam.ac.uk/ gh275/relay.pdf, 2005. 
12. G. P. Hancke and M. G. Kuhn. An RFID distance bounding protocol. In Proceedings of IEEE/Create-Net SecureComm 2005, pages 67-73. IEEE Computer Society Press, 2005.

13. International Rectifier. Data sheet for IRFD110 N-channel MOSFET. http://www.irf.com/product-info/datasheets/data/irfd110.pdf.

14. ISO/IEC 14443. Identification cards - Contactless integrated circuit(s) cards Proximity cards - part 1-4. www.iso.ch, 2001.

15. A. Juels, D. Molnar, and D. Wagner. Security and privacy issues in e-passports. In Security and Privacy for Emerging Areas in Communications Networks, 2005. SecureComm 2005., pages 74-88. IEEE, September 2005.

16. Z. Kfir and A. Wool. Picking virtual pockets using relay attacks on contactless smartcard systems. Cryptology ePrint Archive, Report 2005/052, 2005. http://eprint.iacr.org.

17. I. Kirschenbaum and A. Wool. How to build a low-cost, extended-range RFID skimmer. Cryptology ePrint Archive, Report 2006/054, 2006. http://eprint.iacr.org/.

18. S. Kumar, C. Paar, J. Pelzl, G. Pfeiffer, A. Rupp, and M. Schimmler. How to break DES for 8,980. In International Workshop on Special-Purpose Hardware for Attacking Cryptographic Systems - SHARCS'06, Cologne, Germany, April 2006.

19. T. Lohmann, M. Schneider, and C. Ruland. Analysis of power constraints for cryptographic algorithms in mid-cost RFID tags. In J. Domingo-Ferrer, J. Posegga, and D. Schreckling, editors, Smart Card Research and Advanced Applications, volume 3928 of Lecture Notes in Computer Science, pages 278-288. Springer, 2006.

20. Melexis. Application note: A power booster for the MLX90121. http://www.melexis.com/prodfiles/0003881_AN90121_4_1.pdf.

21. National Semiconductor. Datasheet for LM311 voltage comparator. http://www.national.com/pf/LM/LM311.html\#Datasheet.

22. Y. Oren and A. Shamir. Power analysis of RFID tags. http://www.wisdom.weizmann.ac.il/ yossio/rfid/.

23. Philips. Data sheet for 4 bit binary ripple counter 74393 . http://www.semiconductors.philips.com/pip/74HC393D\#datasheet.

24. Philips. Data sheet for 7408 AND gate. http://www.semiconductors.philips.com/pip/74HC08N.

25. Philips. Data sheet for D type flip-flop 7474 . http://www.semiconductors.philips.com/pip/74F74.html\#datasheet.

26. Philips. Data sheet for monostable multivibrator 74HC/HCT123. http://www.semiconductors.philips.com/pip/74HCT123D\#datasheet.

27. Philips. Data sheet for MIFARE Ultralight Contactless Single-trip Ticket IC. http://www.semiconductors.philips.com, 2003.

28. Philips. Philips scores in German stadiums. On the move, page 3, Mar 2006.

29. M. R. Rieback, B. Crispo, and A. S. Tanenbaum. The evolution of RFID security. Pervasive Computing, 5(1), Jan-Mar 2006.

30. Texas Instruments. HF Antenna Cookbook Technical Application Report. http://www.ti.com/rfid/docs/manuals/appNotes/HFAntennaCookbook.pdf, 2004.

31. Texas Intruments. Texas Instruments to deliver RFID solution for MasterCard PayPass. http://www.ti.com/rfid/docs/news/news_releases/2005/ rel01-17-05a.shtml

32. U. Tietze and C. Schenk. Halbleiter-Schaltungstechnik. Springer, eleventh edition, 2001. 